\title{
Design and Implementation of a Flexible GYRO Stabilized Platform
}

\author{
Zhao $\mathrm{Yao}^{1,2}, \mathrm{~J}$ ie L iu ${ }^{1}$, Yungong $\mathrm{LI}^{1}$, Xaohao $\mathrm{LI}^{1}$ and Zhanyi Zhang ${ }^{1}$
}

${ }^{1}$ School of Mechanical Engineering \& Automation, Northeastern University, Shenyang 110004 Liaoning, China

${ }^{2}$ Army Armor Institute of PLA, Changchun 130117 Jilin, China

Keywords: Gyro stabilized platform; Line of sight stabilization; Sliding mode variable structure control;Flexible gyro.

\begin{abstract}
Aiming at the stability problem of armored equipment firing in advance, a flexible gyro stabilized platform is designed in this paper. The structure of the stabilized platform is introduced, the factors affecting the platform error are analized, and the ideal control strategy is designed. Through the experimental analysis, the platform has better stability.
\end{abstract}

\section{Introduction}

Gyro stabilized optical detection platform as a kind of high accuracy of line-of-sight stabilization system, can provide panoramic range detection and tracking. Both can be used as independent electro-optical observation and aiming system for reconnaissance missions, but also can be used as fire control system components to complete the battlefield combat mission. The important function of isolating the angular motion of a carrier, so that the detector in the inertial space remained stable; in response to a command signal, in a certain range of search on the target, the artificial identification lock according to the detector signal automatic target tracking, azimuth, pitch angle and gives information ${ }^{[1]}$. Improve the stability and accuracy of stabilized platform is aimed at the direction of the line of stable development.

Line of sight stability control key technologies: light reflection mirror design and processing technology, precise transmission organizations design, high-precision target line stability control system design as well as gyro sensors crucial design selection. Strict quality assurance in the manufacturing and assembly process, optimizing the system control law and parameter matching is the main measures to ensure the accuracy of steady aim. This system of flexible gyro feedback angular rate stabilization loop, the use of the torque motor as the actuator to drive the gyro, tops mirrors and solver. mirrors, lightweight titanium alloy supplemented by high-precision processing and debugging process, compared with the traditional framework of gyro-stabilized platform, the load characteristic features, fast response, high stability and accuracy, strong impact and so many advantages.

\section{The Structure of Stabilized Platform}

The stable platform is mainly composed of a biaxial rate integrating gyroscope, azimuth and height to the DC torque motor, azimuth and height to the solver, $1 / 2$ strip transmission mechanism, a supporting frame, an electromagnetic locking device and light reflector. Limited angle torque motor for DC torque motor, mounted on the head of mechanical rotation frame, two motors are respectively provided the elevating axis azimuth axis output torque. Biaxial rate integrating gyro using flexible gyro, installed on the head frame, it is the core component of stable platform, used for sensitive body disturbance rates and mirror rotation angle, through linear change, variable coefficient, and stable servo correction circuit, a power amplifying circuit and torque motor to form a stable, servo control system, realize the reflector on earth space stability. Figure 1 is a block diagram of stable platform. 


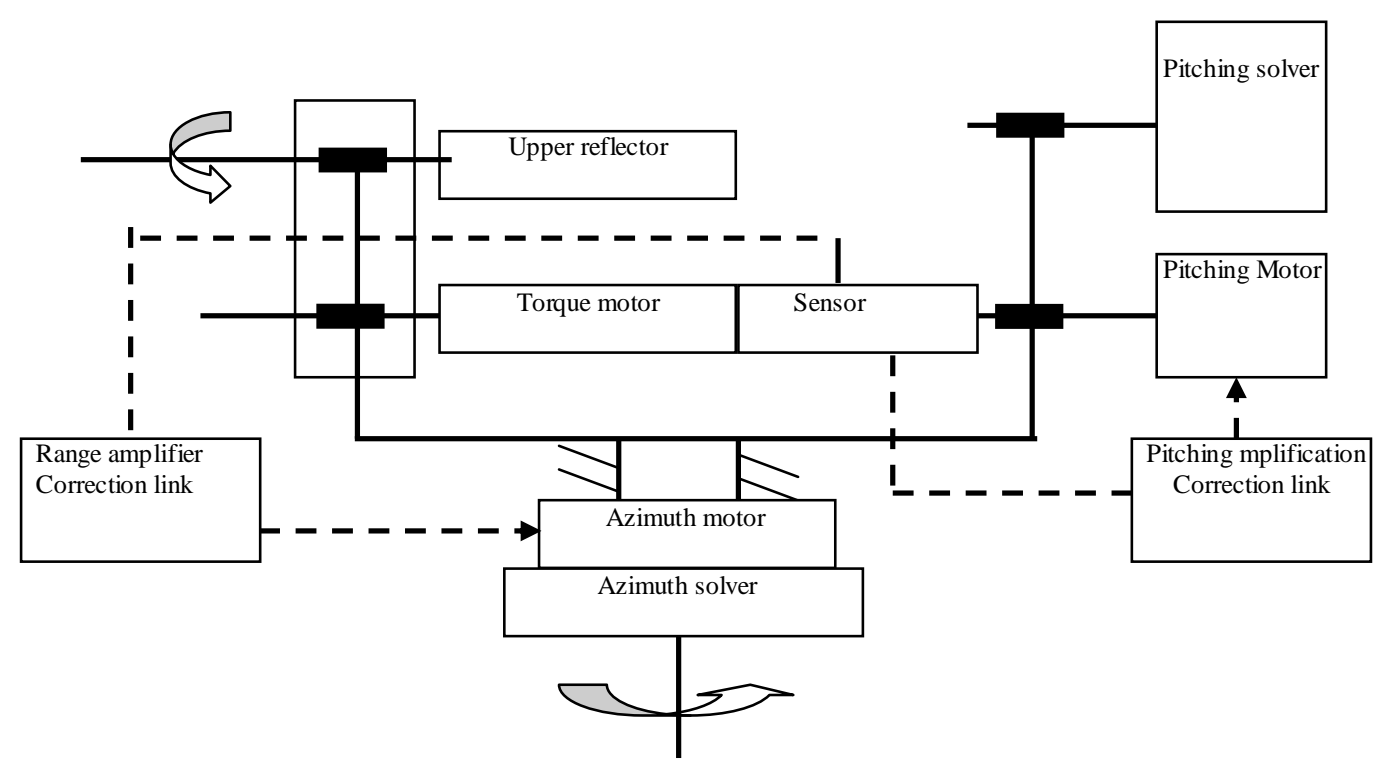

Figure 1. Finite Block diagram of stabilized platform

\section{Overall Design of Stabilized Platform}

The three dimensional structure modeling and analysis software, analysis of the stable platform mechanical structure, transmission structure characteristics and load characteristic parameters, decided to close the heavy sensing device performance parameters. According to the simulation to calculate the load characteristics and the overall system of stabilized sighting system performance indicators, on the counter assembly mounted within the gyro angle sensor, motor and the key components such as selection, the control system modeling, simulation calculation of circuit parameters. At the same time, in order to improve the modeling accuracy, in addition to the use of advanced 3D modeling software accurate simulation calculation on the load characteristic, according to the system requirement and the selected sensor characteristic parameters, the system modeling with Matlib \& Simulink system design and analysis software, consider the various parameter disturbance, model analysis and digital simulation, repeated verification of the accuracy of the model. Considering the stabilized sighting system is a high precision control system, gyro, rotating transformer device output information belongs to the weak signal, are extremely susceptible to electromagnetic interference, affects the system stable precision or synchronization, and system control circuit in high power and high frequency pulse current, the stabilized sighting system impact, therefore, on the system and circuit all weak signal and large current, high frequency pulse signal are done shielding, grounding circuits using the classification of single point grounding and grounding shell, filter circuit also employs a metal shield, to minimize interference [2].

The control module adopts an integrated DC / DC power module partition unified power supply, power supply and high precision, good reliability, good stability; power amplification circuit using current feedback protection mode, and the integration of heat, improve the reliability of the whole system. The position servo control system adopts a digital control mode to realize the position sensor signal acquisition and processing and correction of the position loop, speed using sliding mode variable structure controller adjusts the control.

\section{Precision Influence Factor Analysis of Platform Stabilization}

Gyro stabilized platform as a typical servo motion control system, which has a lot of influence on system performance of nonlinear factors, seriously affecting the static and dynamic property [3]. These factors are mainly divided into the following aspects:

Nonlinearity Error of Flexible Gyro Output. In the line of sight stabilization control, as the inertial space rate sensitive element, the flexible gyro performance to the performance of the whole system plays an important role, its measurement output feedback signal accuracy largely determines the system of LOS 
stabilization accuracy. Flexible gyroscope is fixedly connected directly with the carrier, the carrier angular motion acts directly on the gyroscope, so in addition to carrier line motion caused by disturbance torque, also will appear the angular motion of a carrier induced disturbance torque, plus $\mathrm{V} / \mathrm{F}$ transform nonlinear dead zone, and other factors, so that the flexible gyro output often presents nonlinear. Usually, the gyro scale factor nonlinearity reached about $1 / 1000$, in the high precision requirement is high, the error compensation. In addition the gyro's drift signal and noise by controlling the effects of control loop, thereby forming of LOS stabilization uncertain disturbance, so the output of the gyro drift error and noise suppression is the key to ensure that the visual axis of stability [4].

Mechanical Structure Error. Mainly includes the frame shaft verticality and unbalance degree error. Because the frame centroid deviation from the rotary shaft static unbalance torque error, so that the base of linear vibration on the frame each shaft form a disturbance torque; the cable flexibility will produce unbalance torque; due to mechanical or mechanical installation caused by frame, mirror shaft coupling error and gear itself gap, gyro its disorders and the installation speed sensitive axis verticality and parallelism and platform caused by the non alignment error and so on [5]. The impact of these factors can inhibit or eliminate in the mechanical design, processing and installation

Mechanical Resonance. Drive shaft elastic deformation is caused by mechanical resonance reason, apart from the mechanical engineering design considerations to take compensatory measures, from the angle of control must also be compensated inhibition. Due to the mechanical resonance in the system properties in the performance for the two order links, often uses the series two order notch filters method counteract oscillation link, due to the influence of work environment, system parameter fluctuations occur, including shafting stiffness coefficient and resonant frequency is difficult to accurately predict, therefore cannot accurate compensation.

Friction Torque. The carrier with the stable platform due to the bearing, torque motor brush, a conductive ring arising from the presence of friction. Friction is the system had a greater effect on the nonlinear factor. From the system point of view, the friction nonlinear equivalent actuator dead zone existing in, leading to the formation of the steady state error of the system; from the motion process, mechanical friction causes the system to run at a low speed circumstances" creeping" phenomenon, and even cause the limit cycle oscillation.

In order to suppress the influence of friction, mechanical engineering with the effective elimination of friction caused by crawling and self-excited oscillation method is to cut off the controlled process itself produce self-excited oscillation within the feedback loop, the low friction machine design, lubrication selection as well as by the describing function or related graphic method on the stability of the system and make the system analysis the mechanical stability of system parameters. From control engineering point of view, by using state observer or friction model identification technology to realize torque dynamic compensation, in ensure the stability of the system under the premise, from error and stability of integrated angle to consider [6].

In real systems, due to the friction moment is multivariable, nonlinear dependencies, different conditions have different manifestations, associated with a variety of mechanisms of interaction and the inherent characteristics of moving objects and other reasons, have universal friction model is not possible, so it is difficult to achieve accurate modeling and compensation, using sliding mode variable structure control strategy to compensate for time varying nonlinear friction is a good choice. At the same time, the carrier of low frequency vibration as well as in azimuth, roll and pitch direction angular motion is due to the existence of friction and coupled to the shaft to form stable platform external disturbance torques.

Drive System Error. Mainly includes the motor torque saturation, rigid, input / output nonlinear characteristics, shaft torque coupling, the load changes, the electrical parameters of the fluctuation and the power drive circuit in electronic circuit noise and quantization error. These are the factors affecting system performance can not be ignored.

In order to obtain high stability accuracy, some of these factors can be affected in mechanical design, processing and installation to be suppressed or eliminated, but the system of gyro noise, friction torque and mechanical resonance on a platform stabilizing effect is difficult to restrain, gyro noise will limit the system bandwidth, thus affecting the system quickly sex, existence of friction will affect the system 's low 
speed characteristic, therefore, the effect of these factors must adopt effective control strategies to overcome them.

\section{Sliding Mode Variable Structure Controller Design}

Gyro line of sight stabilization system in addition to having motion control of common mechanical resonance, motor dead-time and limit cycle oscillation, friction torque coupling load change and the electrical parameters of the fluctuation problem outside, flexible gyroscope itself due to torsion of the elastic stiffness, signal device and torque stability and temperature and so on, so the flexible gyro zero temperature drift phenomenon; in addition the gyroscope internal electronic device will produce thermal noise, gyro external analog signal fluctuation, power interference and electromagnetic interference can lead to random noise; in addition signal in transmission, measurement and conversion process may introduce substantial noise, measurement noise can make identification of the model has a higher order to approach the actual system, will reduce the precision of control system and resolution, but, this kind of photoelectric tracking system with a very short reaction time, stable control response time generally at the millisecond level, thus requiring control system has a fast dynamic response, high quality precision as well as for the model parameters adaptive ability and robustness. The conventional PID controller is difficult to achieve the ideal control effect.

Variable structure control system is a special kind of nonlinear feedback control system is an effective method to solve bounded unmeasurable disturbance, the system variable parameter and model uncertainty. Sliding mode can be designed with the object parameters and disturbance has nothing to do, making the sliding mode variable structure control with fast response, parameter variation and disturbance insensitive, without system identification, physical implementation and simple. Its application to the stabilized platform system can solve the contradiction between the accuracy and rapidity, and enhance system robustness, inhibit the disturbance torque of the motor, and the algorithm is relatively simple and works well and easy to implement [7].

The introduction of sliding mode variable structure control system model shown in Figure 2.

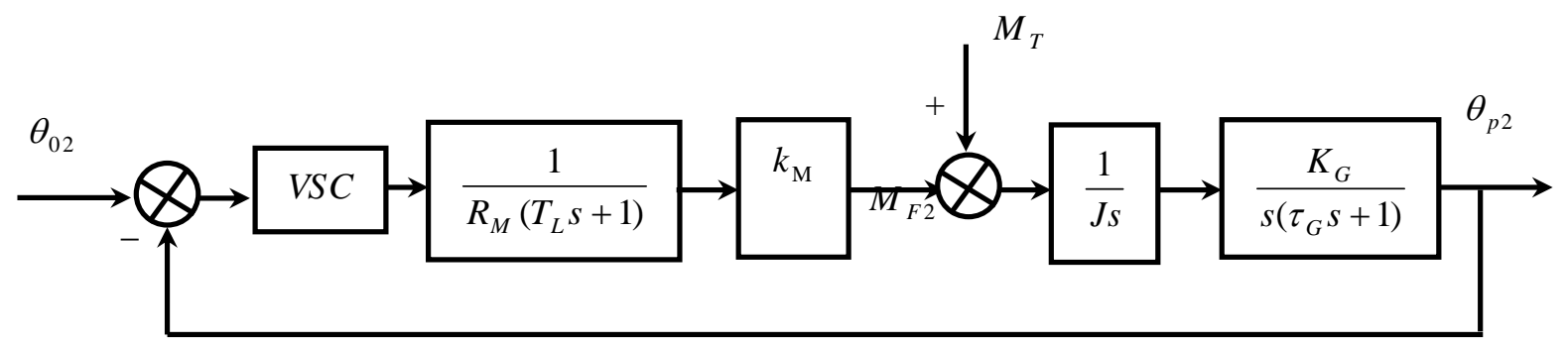

Figure 2. Finite System Model included sliding mode variable structure control

The system transfer function is

$$
G(s)=\frac{12012}{s^{2}(0.012 s+1)}=\frac{1001000}{s^{3}+83 s^{2}}
$$

Discrete state space expression for

$$
x(\kappa+1)=A x(k)+B u(k)=\left[\begin{array}{ccc}
1 & 0.00025 & 0 \\
0 & 1 & 0.0025 \\
0 & 0 & 0.9792
\end{array}\right] x(k)+\left[\begin{array}{c}
0 \\
0 \\
250
\end{array}\right] u(k)
$$


Take

$$
R(k)=\left[\theta_{02}(k) ;+d \theta_{02}(k) ; d^{2} \theta_{02}(k)\right], \quad R(k+1)=\left[\theta_{02}(k+1) ;+d \theta_{02}(k+1) ; d^{2} \theta_{02}(k+1)\right]
$$

The switching function can be written as

$$
s(k)=C e E=C e(R(k)-x(k))
$$

Among them $C e=\left[C_{1}, C_{2}, 1\right]$, then

$$
s(k+1)=C e(R(k+1)-x(k+1))=C e R(k+1)-C e A x(k)-C e B u(k)
$$

Control law is

$$
u(k)=(C e B)^{-1}(C e R(k+1)-C e A x(k)-s(k+1))
$$

Substitution $s(k+1)-s(k)=-q T_{s} s(k)-\varepsilon T_{s} \operatorname{sgn}(s(k))$, then

$$
u(k)=(C e B)^{-1}\left(C e R(k+1)-C e A x(k)-s(k)+\varepsilon T_{s} \operatorname{sgn}(s(k))+q T_{s} s(k)\right)
$$

Where $c_{1}, c_{2}, q, \varepsilon$ are the variable parameters. Sliding mode parameters $c_{1}$ and $c_{2}$ has great effect on adjusting time. The main parameters $q$ influencing the reaching speed switching function for dynamic switching process, appropriate adjustment of the parameters to change the system to the sliding mode surface reaching speed, can better improve the dynamic performance of the system. Symbol function gain parameter represents the system to overcome the perturbation and external disturbance ability [8].

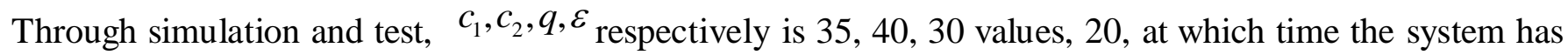
smaller steady-state error and anti jamming ability.

\section{Test and Results Analysis}

On the stabilization of sight system were to do the swing test, vibration test and impact test. Test, in the main test platform (swing, vibration, shock ) placed above the center position of a plane mirror, the stabilized platform is fixed on the main test bench, and the stable platform bottom axial plane mirror axial coincidence. Parallel optical tube is used to measure the component on the mirror movement, light tube eyepiece to is central to the origin, have a certain scale and below[9]. On the parallel light pipe power, make it to the front emitting parallel light beam, the eyepiece alignment measuring component on the mirror, while on the mirror will be parallel to the beam of light to a plane mirror; then the beam will be along the same path to return to the parallel light pipe to the eyepiece, forming a cross partition, by adjusting the eyepiece in the azimuth and elevation the axial position, can make a crosshair reticle eyepiece origin coincident with. In experiment, observation eyepiece in the cross divisional trajectories, knowable stabilized platform height to the stable error and azimuth stabilization error epsilon beta, the specific data such as shown in Table 1 to Table 3. 
Table 1 Stabilization error of swing test

\begin{tabular}{|c|c|c|c|}
\hline \multirow{2}{*}{ Test conditions } & Steady state error & $\varepsilon$ & $\beta$ \\
\hline \multirow{2}{*}{$\begin{array}{c}\text { The level of } \\
\text { direction }\end{array}$} & $2.5^{\circ} / 0.8 \mathrm{~Hz}$ & $30 "$ & $20^{\prime \prime}$ \\
\cline { 2 - 4 } & $6 \% 1 \mathrm{~Hz}$ & $1^{\prime} 10^{\prime \prime}$ & $40^{\prime \prime}$ \\
\cline { 2 - 4 } & $10^{\circ} / 1 \mathrm{~Hz}$ & $1.20^{\prime \prime}$ & $1^{\prime}$ \\
\hline \multirow{2}{*}{$\begin{array}{c}\text { The horizontal } \\
\text { direction }\end{array}$} & $10.8^{\prime} / 3 \mathrm{~Hz}$ & $40^{\prime \prime}$ & $0^{\prime \prime}$ \\
\cline { 2 - 4 } & $0.5^{\circ} / 1 \mathrm{~Hz}$ & $10^{\prime \prime}$ & $40^{\prime \prime}$ \\
\cline { 2 - 4 } & $2 \% 0.8 \mathrm{~Hz}$ & $20^{\prime \prime}$ & $30^{\prime \prime}$ \\
\hline Heeling direction & $2.5^{\prime \prime} / 1 \mathrm{~Hz}$ & $20^{\prime \prime}$ & $50^{\prime \prime}$ \\
\hline
\end{tabular}

Table 2 Stabilization error of vibration test

\begin{tabular}{|c|c|c|c|}
\hline \multicolumn{2}{|c|}{ Steady state error } & $\varepsilon$ & $\beta$ \\
\hline \multirow{4}{*}{ The amplitude of the $0.2 \mathrm{~mm}$} & $10 \mathrm{~Hz}$ & $10 "$ & $10 "$ \\
\hline & $20 \mathrm{~Hz}$ & $30 "$ & $15^{\prime \prime}$ \\
\hline & $30 \mathrm{~Hz}$ & $10^{\prime \prime}$ & $20 "$ \\
\hline & $40 \mathrm{~Hz}$ & $20 "$ & $20 "$ \\
\hline \multirow{7}{*}{ 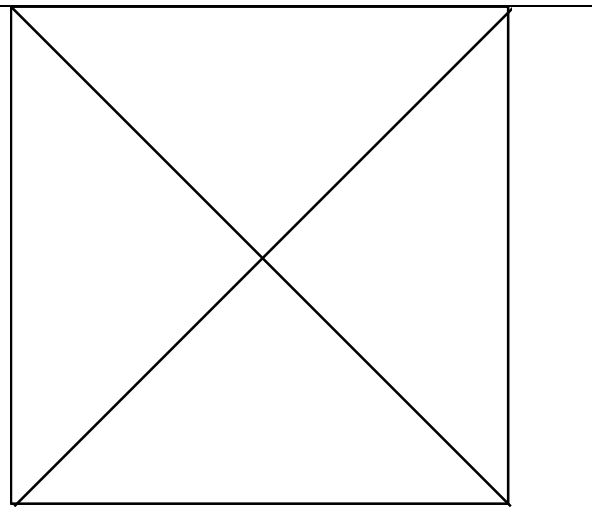 } & $50 \mathrm{~Hz}$ & $40 "$ & $40 "$ \\
\hline & $60 \mathrm{~Hz}$ & $15^{\prime \prime}$ & $45^{\prime \prime}$ \\
\hline & $70 \mathrm{~Hz}$ & $30 "$ & $1^{\prime}$ \\
\hline & $80 \mathrm{~Hz}$ & $1^{\prime}$ & $1^{\prime} 20^{\prime \prime}$ \\
\hline & $90 \mathrm{~Hz}$ & $20 "$ & $50 "$ \\
\hline & $100 \mathrm{~Hz}$ & $10^{\prime \prime}$ & $40 "$ \\
\hline & $110 \mathrm{~Hz}$ & $20 "$ & $30 "$ \\
\hline $\begin{array}{l}\angle \\
\text { Acceleration of } 2 \mathrm{~g} \text {, each } 10 \mathrm{~Hz} \text { is a } \\
\text { section, each section of } 2 \mathrm{~min}\end{array}$ & $120 \mathrm{~Hz}$ & $20 "$ & $1^{\prime}$ \\
\hline
\end{tabular}

Table 3 Stabilization error of impact test

\begin{tabular}{|l|l|l|}
\hline \multicolumn{1}{|c|}{ Steady state error } & \multicolumn{1}{|c|}{$\varepsilon$} & \multicolumn{1}{|c|}{$\beta$} \\
\hline $\begin{array}{l}\text { Half sine wave, continuous } \\
6 \mathrm{~ms} \text {, peak acceleration 30g, } \\
\text { along the line of sight } \\
\text { direction }\end{array}$ & $\begin{array}{l}\text { Impact of line of sight } \\
\text { around 4' after impact, line } \\
\text { of sight the variational } \\
\text { momentum of the zero } \\
\text { position is 10" }\end{array}$ & $\begin{array}{l}\text { Impact of line of sight around 2 after } \\
\text { impact, line of sight the variational } \\
\text { momentum of the zero position is 0 }\end{array}$ \\
\hline $\begin{array}{l}\text { Half sine wave, continuous } \\
\text { 3ms, the peak acceleration of } \\
50 \mathrm{~g}, \text { along the line of sight } \\
\text { direction }\end{array}$ & $\begin{array}{l}\text { Impact of line of sight } \\
\text { around 5' after impact, line } \\
\text { of sight the variational } \\
\text { momentum of the zero } \\
\text { position is 20" }\end{array}$ & $\begin{array}{l}\text { Impact of line of sight around 3 } \\
\text { after impact, line of sight the } \\
\text { variational momentum of the zero } \\
\text { position is 0 }\end{array}$ \\
\hline
\end{tabular}

Through analyzing the experiment data can reach the following conclusion: 
( 1 ) the stable platform of static stability accuracy of no more than 10 "; in the use of parallel light pipe for static observation, line of sight remained motionless, static accuracy far exceeds the requirements of noise suppression system, proved more successful, can achieve the basic no static error.

(2) The stable platform realizes the speed feedback of angle without static error of gyroscope stabilization loop, together with pre filter correction link is introduced, eliminating the closed-loop zeros, reached a better dynamic performance, reliable work, stable and high precision, stable platform for precision reaches the design requirements.

(3)In whole azimuth angle range, line of sight along the azimuth amplitude, in $10 \sim 40 \mathrm{~Hz}$ frequency range, no more than $20^{\prime \prime}$, in $40 \sim 120 \mathrm{~Hz}$ frequency range, no more than $30^{\prime \prime}$. But in $40 \sim 70 \mathrm{~Hz}$ resonant frequency point of $1^{\prime}$; in $70 \sim 120 \mathrm{~Hz}$ resonant frequency point of $2^{\prime}$. This is caused by mechanical resonance. System wide band mechanical resonance influence on system's dynamic performance is more significant. So in the machining process, should try to improve the system of the mechanical resonant frequency, in order to make it far away from the system in the band. For the suppression of mechanical resonance frequency, in the acquisition of gyro data amplification correction pretreatment using a notch filter, in $120 \mathrm{~Hz}$, vertical and horizontal did not appear serious ultra almost, within $40 \mathrm{~Hz}$, system errors are less than 20", proved the system low frequency with high precision.

(4) The influence on system accuracy of gun recoil of the most severe, impact test proved this point. In the process of impact, gyro output signal is very large, in the 50g peak acceleration, maximum system dynamic error of up to 5 ', but in shock after, line of sight can be basic regression in situ, the system to meet the design requirements.

\section{References}

[1] HAN Yong-gen. Design and Implementation of a Gyro Stabilized Platform with Zero Steady-state Angle Error[J]. Opto-Electronic Engineering, 2008, 35 (8) : 1 4.

[2] ZHANG De-ning. Research on Digital Control of Upper Reflector Aim-Stabilization System[D]. 2006

[3] Jrtega. Gunfire Performance of Stabilized Electro-optical Sights[C]. Orlando: SPIE Conf, 1999, 3692: 74-83.

[4] LI Han-zhou, LIU Xiu-yan. Error compensation technique for SINS with dynamically tuned gyroscope [J]. Journal of Chinese Ineaial Technology，2007，15（4） : 403-411.

[5] He Debao.The Assembly and Adjustment Technology of the Four-Axis Flexible Gyro Stabilized Platform[J]. M ISSILES AND SPACE VEH ICLES, 1996, 224: 23-27.

[7] LIU Qiang, ER Lian-jie, LIU Jin-kun. Overview of characteristic, modeling and compensation of nonlinear friction in servo system[J]. System Engineering and Electronics, 2002, 24(11): 45-52.

[8] Guo Guilian, Sheng Guangzhong, Cui Zhiqiang. Full-Digital SM VSC DC Servo System Based on DSP[J]. Journal of China Three Gorges University. (Natural Science Edition), 25 ( 5) : 437-439.

[9] Yao Qionghui, Huang Jiqi, Wu Hansong, VSC Control System. Chongqing: Press of Chongqing University, 1997: 1 12 